\title{
Kamelien, Kamelienroute und Pazo da Saleta in Galicien
}

\author{
Hilke Steinecke \& Silvia Rodríguez Coladas
}

\begin{abstract}
Camellias cherish the mild and humid climate of the Atlantic coast of Galicia (northern Spain). They were introduced there around the end of the 18th century. They were initially cultivated especially in parks of castles and manors (pazos), with beautiful, older and splendid specimens still existing today. The Galician „Camellia Route" encompasses 12 gardens with many and particularly attractive camellias. The private Pazo da Saleta is accessible by appointment.
\end{abstract}

\section{Zusammenfassung}

In Galicien/Nordspanien bietet das mild-feuchte atlantische Klima ideale Wachstumsbedingungen für Kamelien. Diese kamen etwa Ende des 18. Jahrhunderts nach Galicien. Sie wurden damals vor allem in Parks von Schlössern und Herrenhäusern (Pazo) gepflanzt, in denen heute z. T. noch sehr alte und prächtige Exemplare stehen. In Galicien wurde aus touristischen Gründen die Kamelienstraße ausgewiesen, zu der zwölf verschiedene Gärten mit besonders schönen, vielen und alten Kamelien gezählt werden. Einer dieser Gärten gehört zum privaten Pazo da Saleta. Dieser Garten kann nach Absprache besichtigt werden und wird hier vorgestellt.

\section{Kamelien - Exotische Schönheiten aus fernem Asien}

Kamelien (Camellia) gehören wie auch der Echte Tee (Camellia sinensis) zu den Teegewächsen. Die Gattung umfasst je nach Auffassung zwischen 200 und 300 Arten. Während der Teestrauch nur relativ kleine, weiße Blüten entwickelt, erreichen Kamelienblüten Durchmesser bis zu $15 \mathrm{~cm}$. Meist sind sie weiß, rosa oder rot gefärbt, seltener gibt es auch gelb blühende Arten wie z. B. Camellia nitidissima. Die in China heimische gelbe Kamelie wurde in Europa erst in den 1970er-Jahren bekannter. Ihre immergrünen Blätter erinnern etwas an Kaffeeblätter, die nickenden Blüten haben wachsig-lederige Konsistenz.

Die ersten Kamelien gelangten vor rund 300 Jahren wahrscheinlich eher ungewollt nach Europa. Vermutlich wurde die Teepflanze mit der Kamelie verwechselt oder sogar bewusst statt der Teepflanze den Kaufleuten „untergejubelt“. Eine anfängliche Enttäuschung über die vermeintlich, nicht aromatische Teepflanze wurde sicherlich schnell durch den hohen Zierwert der Kamelien ausgeglichen. Während $\mathrm{Ka}-$ melien bei uns nur bedingt winterhart sind, wachsen sie in wintermilden, feuchten Regionen wie an der Atlantikküste in Nordwest-Europa (Portugal, Spanien) sowie in Großbritannien sehr gut. Besonders in Galicien lassen ein feuchtes Klima, milde Temperaturen und ein saurer, fruchtbarer Boden Kamelien besonders üppig gedeihen.

Portugiesische Seefahrer brachten Kamelien aus der in Südchina liegenden portugiesischen Kolonie Macao nach Europa. Nach Galicien kamen die Kamelien etwa seit Ende des 18. Jahrhunderts. Da die Kamelien mit ihren auffälligen Blüten anfangs sehr wertvoll und teuer waren, wurden sie zunächst vor allem nur in Parks und Gärten von Schlössern und Herrenhäusern gepflanzt.

Die für Galicien typischen Herrenhäuser, meist auf dem Land gelegen und mit großem Park ausgestattet, werden Pazo genannt. Diese Bezeichnung leitet sich von der lateinischen Vokabel palatium bzw. Palast ab. In manchen dieser historischen Parks der Pazos gibt es heute noch einen sehr alten, beeindruckenden Gehölzbestand, u. a. mit über 200 Jahre alten, einigen Metern hohen Kamelien, die dann eher den Habitus eines kleineren Baumes statt eines Strauches einnehmen.

\section{Die Straße der Kamelien}

Um die Kamelienvielfalt in Galicien bekannter zu machen, wird seit einigen Jahren in Galicien die Kamelienstraße (Ruta de Camelia) beworben und durch Neuanpflanzungen weiter ausgebaut. Bisher sind die galicischen Gärten als 


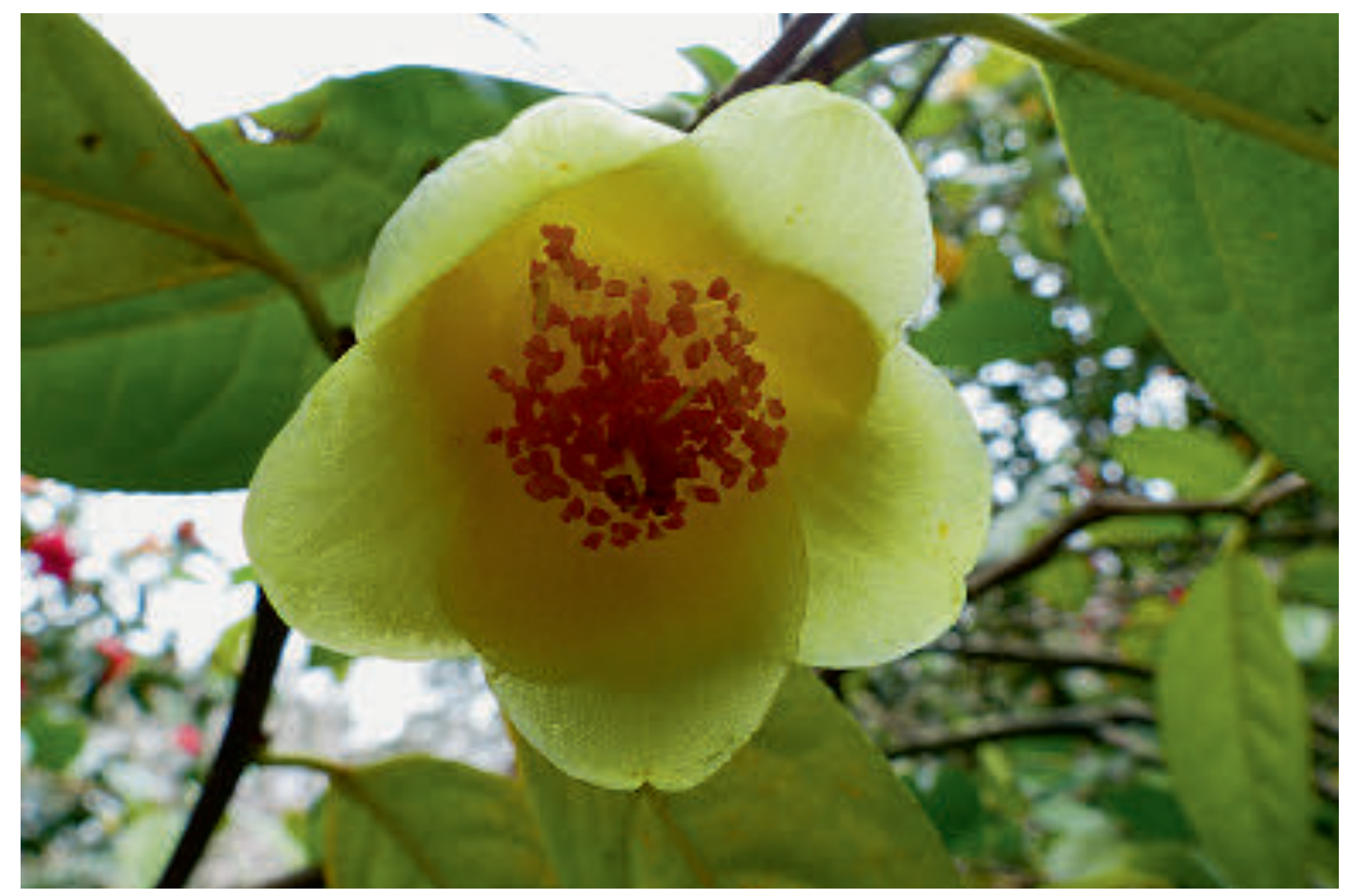

touristische Ziele nur wenig bekannt. Der Tourismusverband Turgalicia und die Spanische Kameliengesellschaft haben auf dieser Route zwölf private und öffentliche Gärten als spezielle Kameliengärten mit einem vielfältigen und z. T. alten Bestand ausgewiesen. Zu jedem dieser Gärten wurde ein zweisprachiger (spanisch/ englisch) Informationsflyer herausgegeben.

In Galicien werden angeblich rund 8000 verschiedene Sorten von Kamelien kultiviert. Es sind Züchtungen der Arten Camelia sasanqua, williamsii-Hybriden und vor allem Sorten von $C$. japonica sowie der besonders großblütigen $C$. reticulata. Alle Gärten aufzusuchen wird man auf einer einzigen Reise wohl kaum schaffen: Wer sich aber einen Überblick über die Vielfalt der Kameliensorten in Galicien verschaffen möchte, sollte die in Galicien jährlich im Wechsel in verschiedenen Orten (z. B. in der hübschen alten Provinzhauptstadt Pontevedra) stattfindende internationale Kamelienausstellung besuchen. Als regelmäßiger Besucher von Kamelienausstellungen im Palmengarten oder anderen deutschen Parks- und Gärten ist man allerdings zunächst sicherlich erstaunt. In der

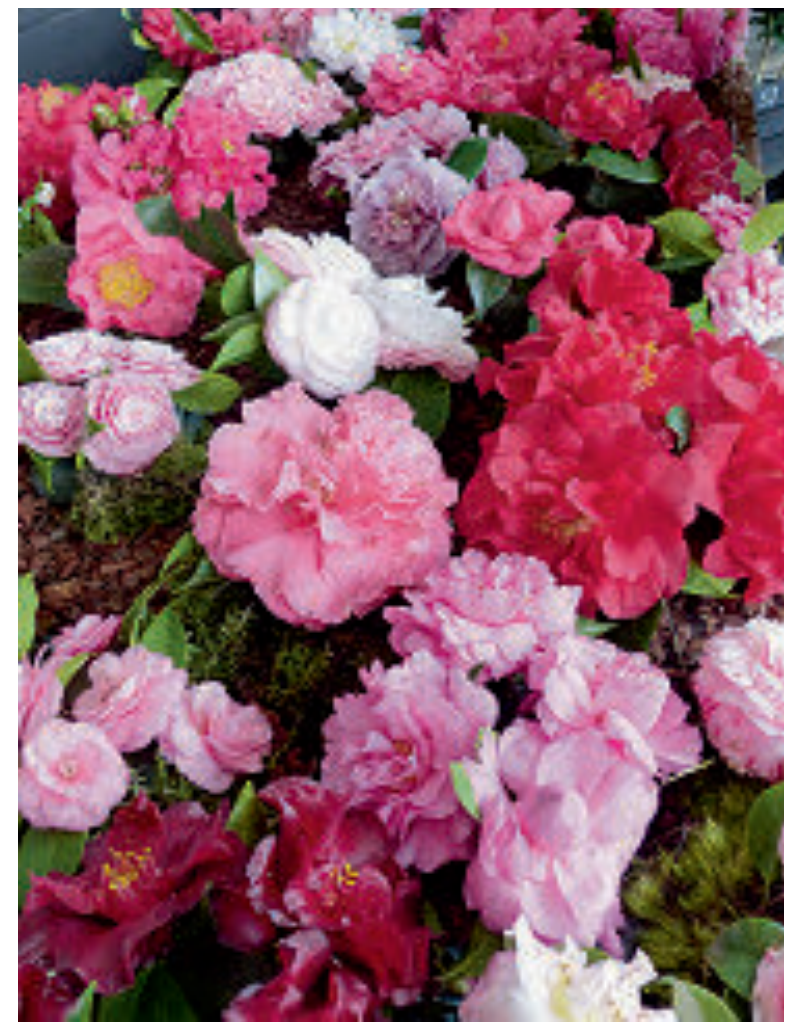

Abb. 1 (oben): Camellia nitidissima ist eine der wenigen gelb blühenden Kamelien.

Abb. 2 (unten): Blütenvielfalt in der Kamelienausstellung in Pontevedra im März 2016. 


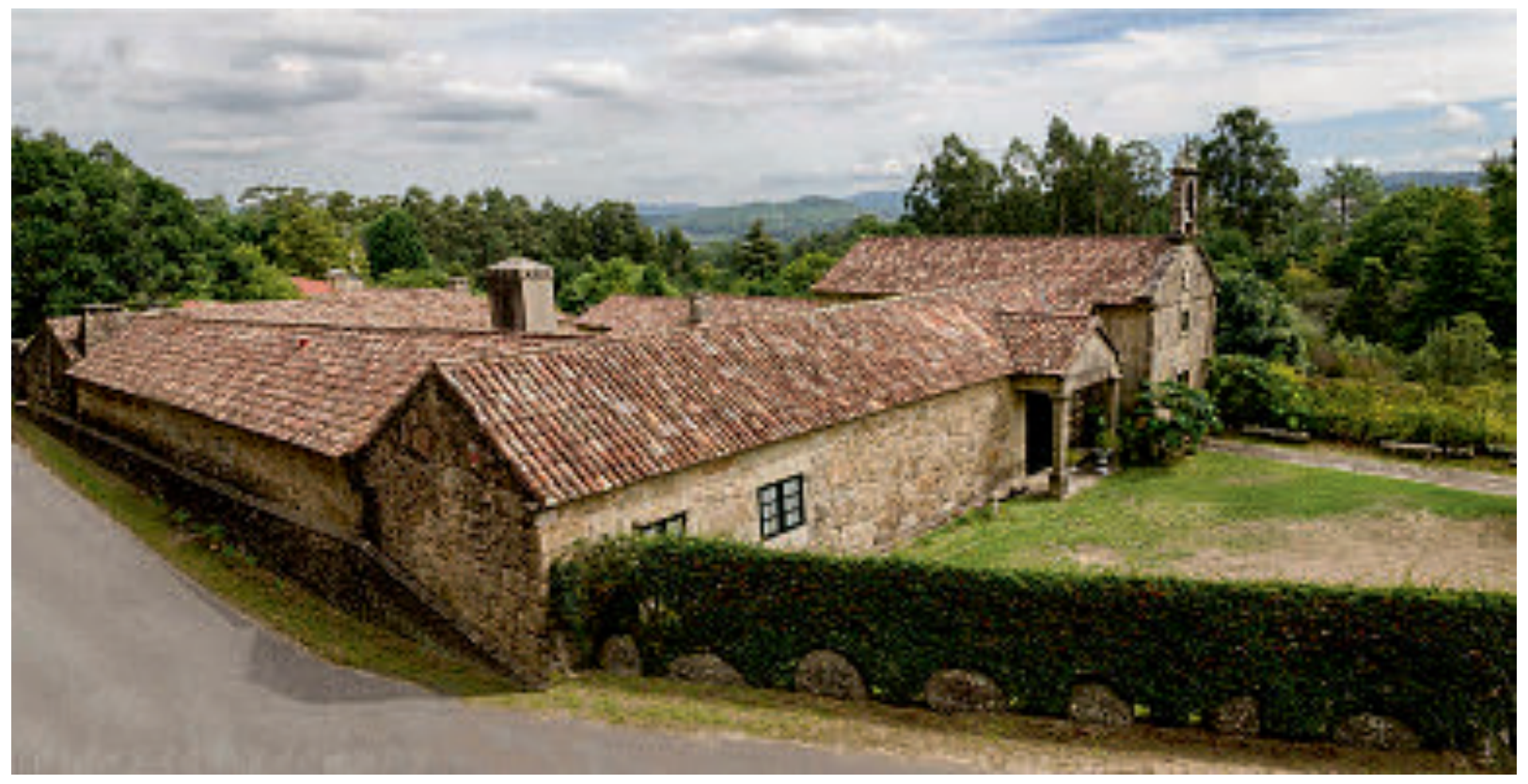

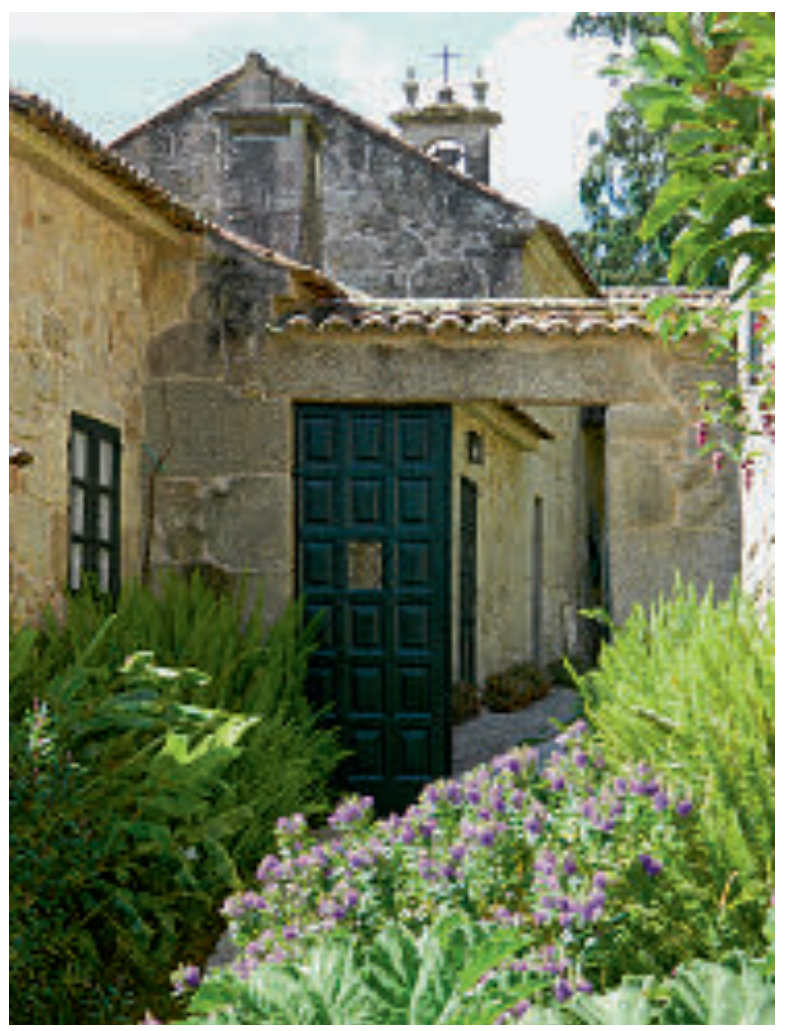

Kamelienausstellung werden nämlich keine ganzen Pflanzen gezeigt (da sie ja vor Ort ausgepflanzt sind und nicht in transportablen Kübeln kultiviert werden), sondern einzelne Blüten der verschiedensten Sorten, die auf Dutzenden von Metern langen Regalen ausgelegt werden. Dementsprechend kann eine derartige Kamelienausstellung auch nicht viel länger als ein bis zwei Tage dauern, da danach die Blüten

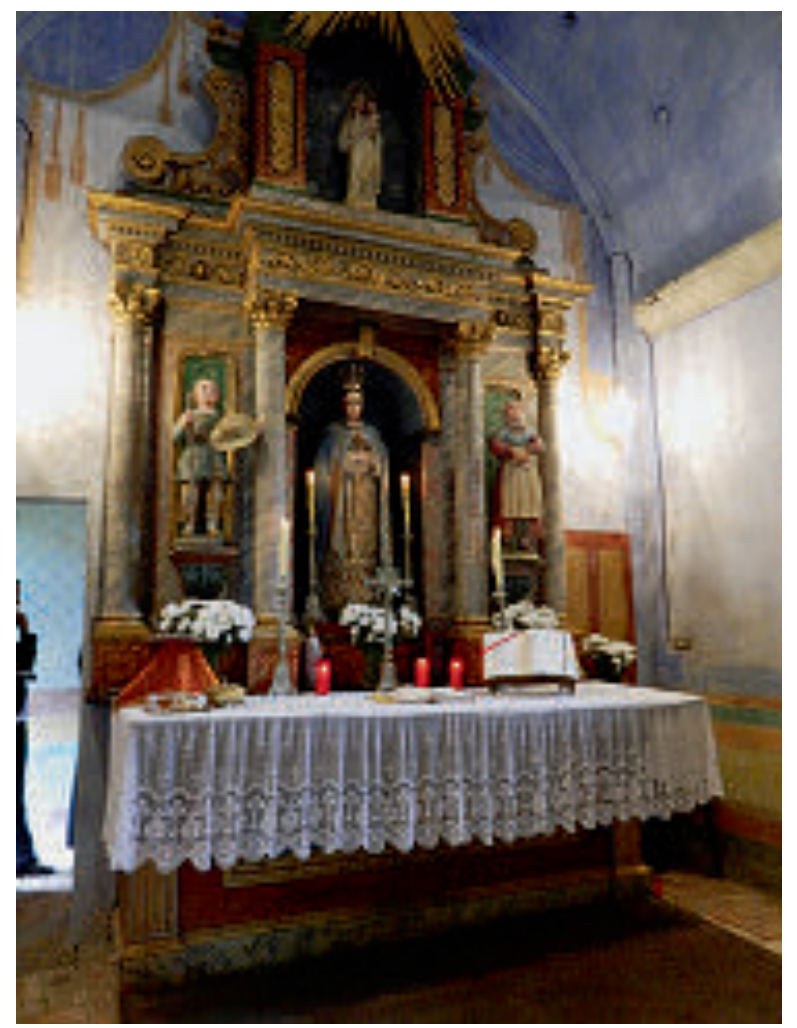

bereits Welkeerscheinungen zeigen. Es lohnt auf jeden Fall, bei der Planung einer Kamelienund Gartenreise diesen Termin zu beachten und gegebenenfalls mit einzuplanen.

Die zwölf, alle im Stil unterschiedlichen Gärten der Kamelienroute sind Pazo de Mariñán, Alameda de Santiago de Compostela, Pazo de Santa Cruz de Ribadulla, Pazo de Oca, CasaMuseo Rosalía de Castro, Pazo de Rubiáns, 
Pazo Quinteiro da Cruz, Pazo de Lourizán, Parque Botánico do Castelo de Soutomaior, Parque do Castro, Xardín do Pazo Quiñones de León und Pazo da Saleta. Kurze Beschreibungen und Lage aller Gärten sind im Internet zu finden. Letztgenannter Garten soll nachfolgend näher vorgestellt werden.

\section{Kurze Geschichte von Pazo da Saleta}

Pazo da Saleta befindet sich in Meis in der Provinz Pontevedra. Auch wenn das Anwesen als Pazo bezeichnet wird, entspricht das Gebäude nicht unbedingt einem typischen galicischen Palast. Es handelt sich eher um ein Landgut, das durch seine frühere landwirtschaftliche Nutzung geprägt ist. Im Jahr 1721 erbte Don Mateo Pérez de Caamaño ein Grundstück und baute darauf einen Hof, den Grundstock von Pazo da Saleta. Einer seiner Nachfahren (Colonel Severo Perez Cardecid) errichtete, vielleicht sogar auf dem Grundriss einer zuvor dort existierenden kleinen Kirche, zwischen 1863 und 1870 eine Kapelle. Sie wurde nach der Jungfrau von Saleta benannt. Im Innern hat die Kapelle einen wertvollen, farbenfrohen Holzaltar, der von dem Künstler Manuel Magasinas aus Santiago de Compostela geschaffen wurde. Bezeichnenderweise sind die Sterne am Himmel der Kapelle achtstrahlig, wie es auch die Sternabzeichen eines Colonels in Spanien sind. Einmal im Jahr, nämlich am Weißen Sonntag, wird - wie früher auch - heute wieder in der Kapelle eine öffentliche Messe gefeiert.

Der Colonel nutzte Pazo da Saleta landwirtschaftlich, während er seinen Hauptwohnsitz in einem anderen Pazo in Cambados hatte. Nach seinem Tode erbte eine seiner beiden Töchter den Hof. Von 1900 - 1936 war Pazo da Saleta dann an den spanischen Staat vermietet,

Abb. 3 (Seite 112 oben): Wohngebäude und Kapelle von Pazo da Saleta.

Abb. 4 (Seite 112 links): Eingangsbereich.

Abb. 5 (Seite 112 rechts): Blick in die Kapelle.

Abb. 6 (oben): Die beiden Besitzerinnen

Silvia Rodríguez Coladas und

Blanca Coladas Carballosa.

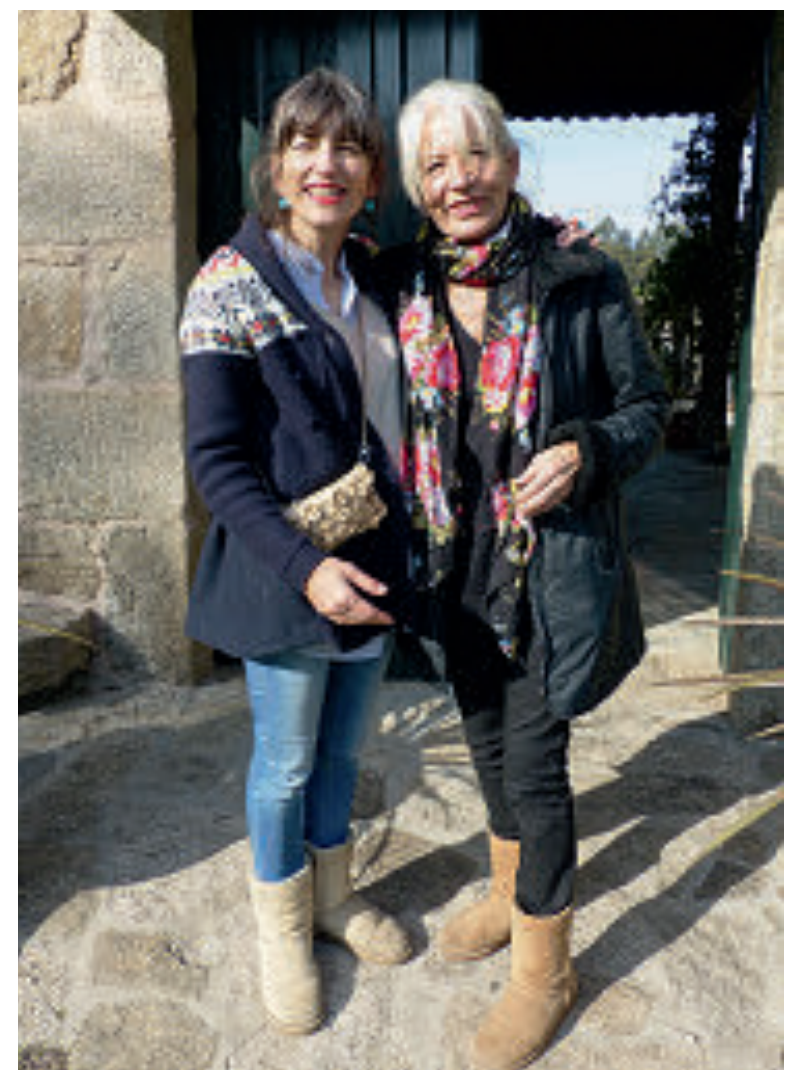

der hier eine landwirtschaftliche Versuchseinrichtung betrieb.

Für die Entstehung des Gartens war es entscheidend, dass im Jahr 1967 das wohlhabende britische Ehepaar Gimson Pazo da Saleta kaufte. Robert Gimson konnte hier seinem Hobby der Botanik nachgehen und sich seinen Traum erfüllen, einen englischen Landschaftsgarten anzulegen. Er wollte nur das Beste vom Besten und konnte die in England hoch angesehene Landschaftsarchitektin BRENDA Colvin für sein Projekt gewinnen. Zusammen mit ihr und seiner Frau Margaret wandelte Robert Gimson das insgesamt 6 ha große Grundstück in einen englischen Landschaftspark mit Charakter eines botanischen Gartens um. Gimsons Vorliebe galt den Kamelien, von denen er zahlreiche Sorten pllanzte. Heute stehen im Garten etwa 200 verschiedene Kamelien. Sammelschwerpunkt waren zudem Pflanzen aus aller Welt, bevorzugt von der Südhalbkugel. Da Gimson mit vielen Gärten im regelmäßigen Austausch stand, erhielt er u.a. auch Saatgut von Raritäten, die in europäischen Gärten nicht überall zu finden sind. Nach seinem Tode sollte 

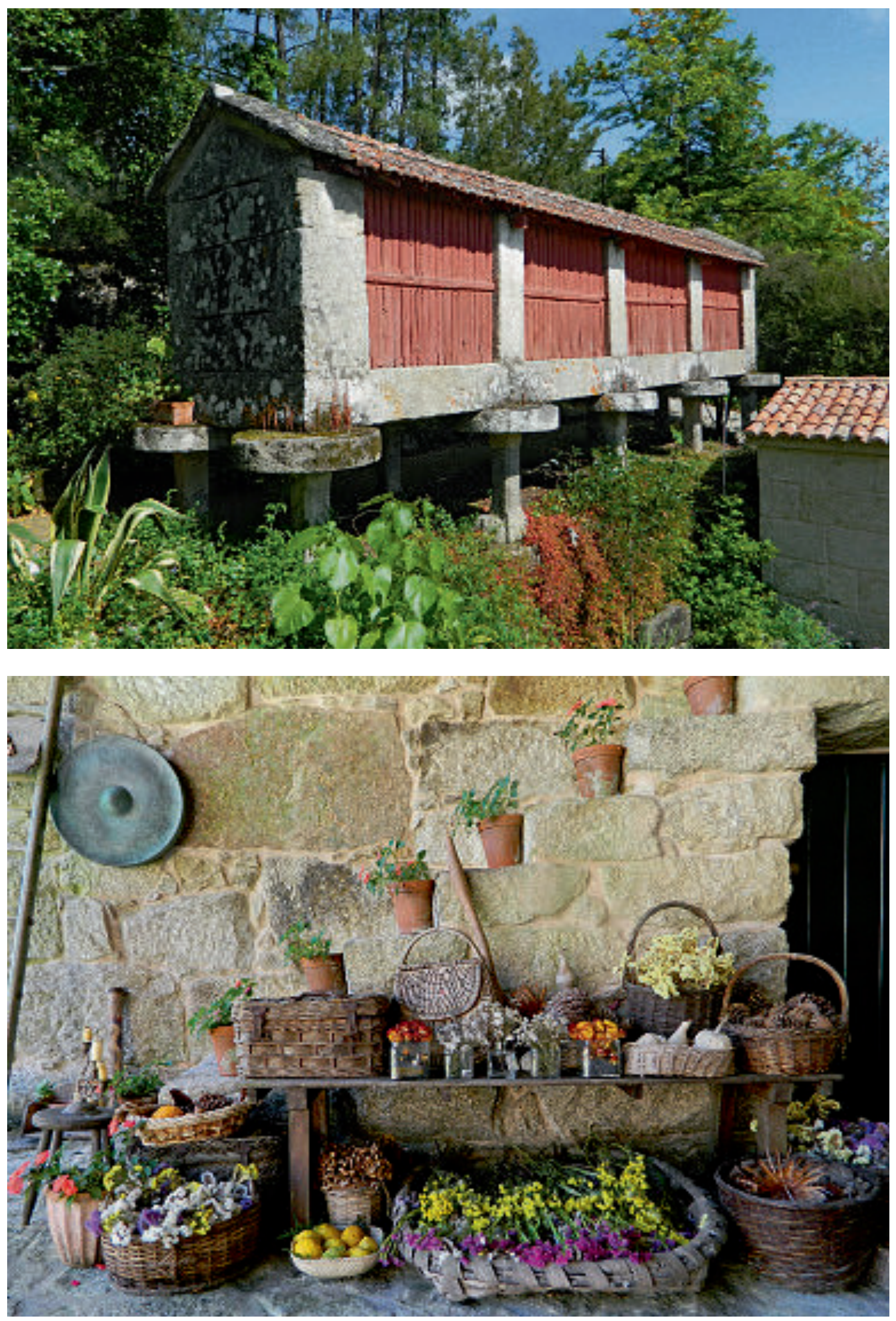
Pazo da Saleta mitsamt dem kompletten Grundstück verkauft werden. Der Preis war jedoch so hoch, sodass sich kein Käufer fand. Schließlich wurde Pazo da Saleta in vier Teile aufgeteilt und diese jeweils an Private veräußert.

Im Jahr 1996 kauften die Zweitautorin und ihre Mutter Blanca Coladas Carballosa den historisch wichtigsten, 2 ha großen Teil mitsamt der Gebäude inklusive des Parks. Nur dieser Teil des ursprünglichen Anwesens kann heute noch besichtigt werden und nur hier lebt die Tradition weiter, die botanische Vielfalt des Parks zu erhalten oder sogar zu erweitern.

\section{Spaziergang durch Pazo da Saleta}

Mit Pazo da Saleta haben sich die beiden Besitzerinnen ein ganz besonderes Paradies geschaffen. Alles ist sehr gepflegt und die aus Natursteinen bestehenden Gebäude sind in einem sehr guten Zustand. Das war nicht immer so: Als Familie Coladas Pazo da Saleta kaufte, war die Kapelle ziemlich verfallen und musste renoviert werden. Den Besitzerinnen ist es ein wichtiges Anliegen, das historische Erbe zu bewahren. Der Park wird gepflegt, die Pflanzen werden erhalten und wenn immer es nötig oder möglich ist, werden Pflanzenspezialitäten aus aller Welt nachgepflanzt. Der Pflanzenbestand ist sehr gut dokumentiert und nahezu jede Pflanze trägt ihr Namensetikett. Der Garten von Pazo da Saleta umfasst aktuell eine der größten privaten Pflanzensammlungen Spaniens.

Eine vielfältige exotische Pflanzenwelt trifft hier auf kleine liebevolle künstlerische Installationen aus der Hand der Besitzerin, was dem Garten einen ganz besonderen Charme verleiht. Auf breite Wege im Garten wurde verzichtet,

Abb. 7 (Seite 114 oben): Traditioneller Speicher (Horreo).

Abb. 8 (Seite 114 unten): Dekoration im Bereich der Scheune.

Abb. 9 (oben): Wasserhahn mit gläsernem Tropfen.

Abb. 10 (Mitte): Watsonien hinter dem Haus geben dem Garten ein feuriges Erscheinungsbild.

Abb. 11 (unten): Aloe plicatilis.
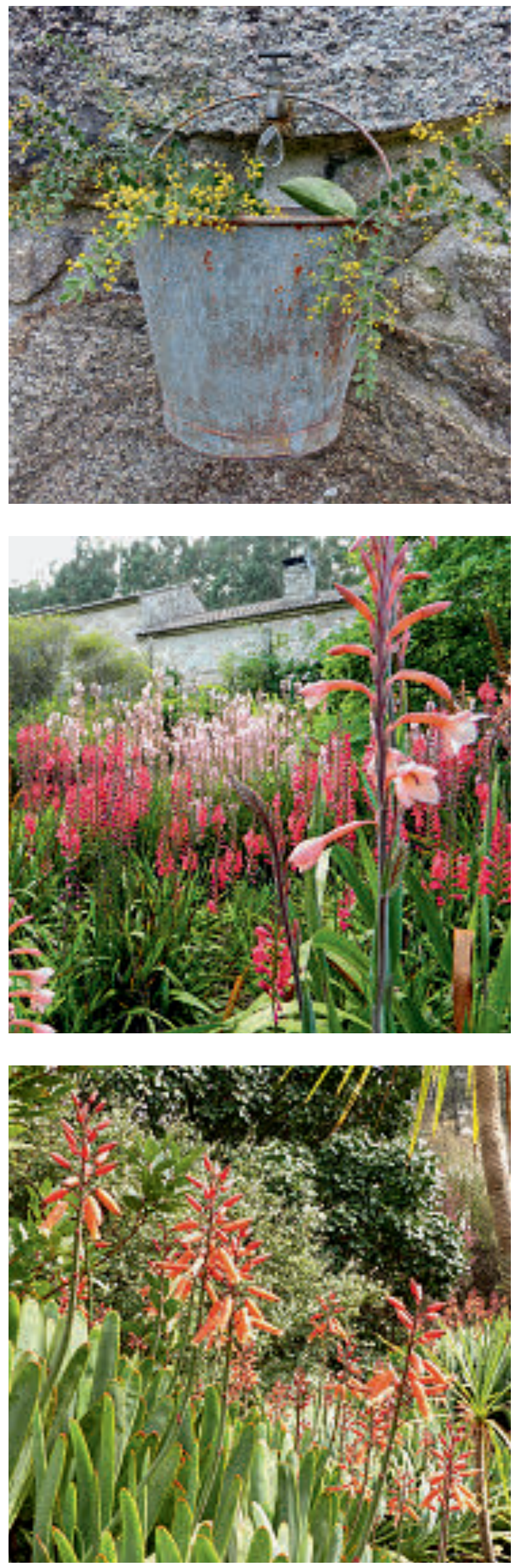

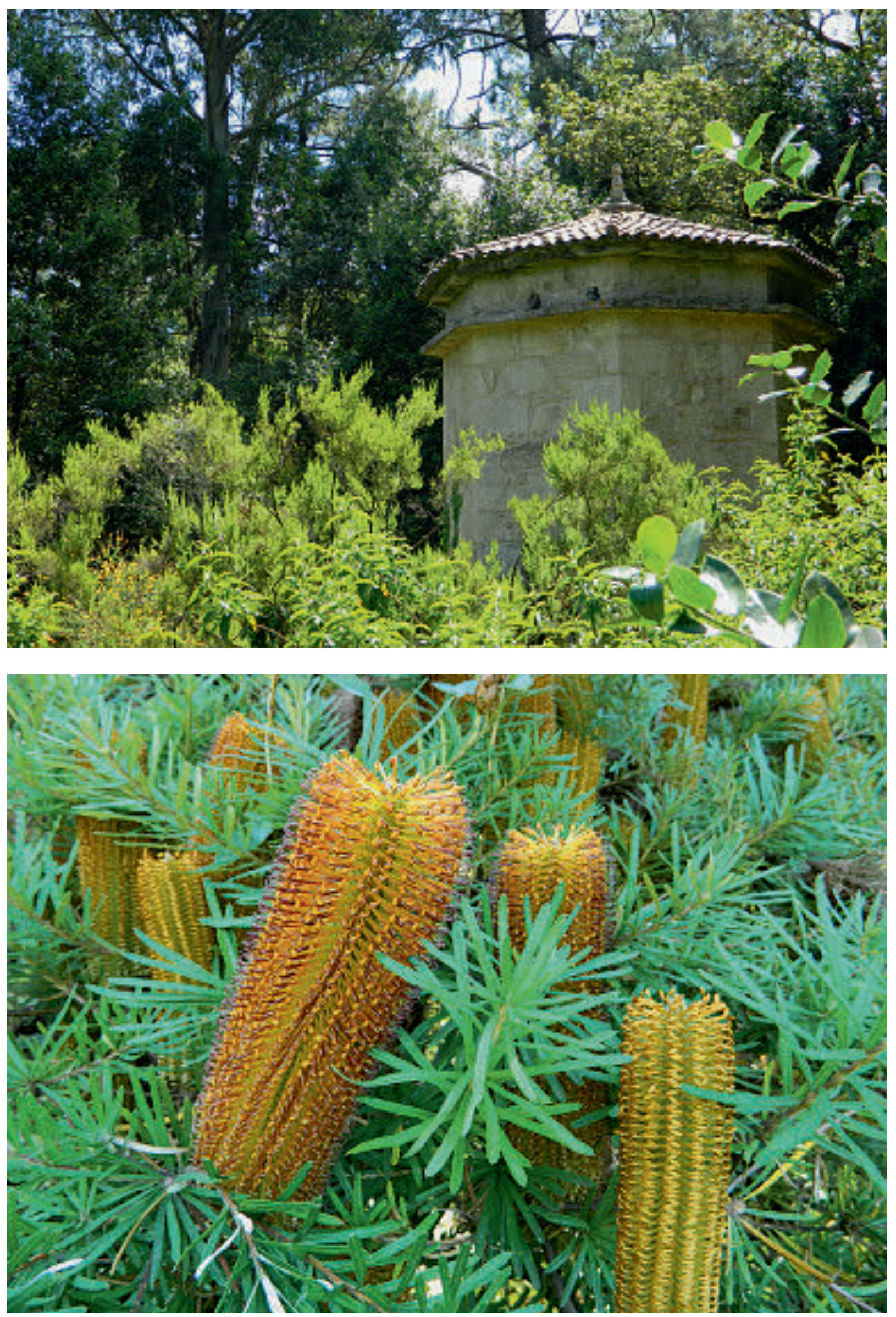


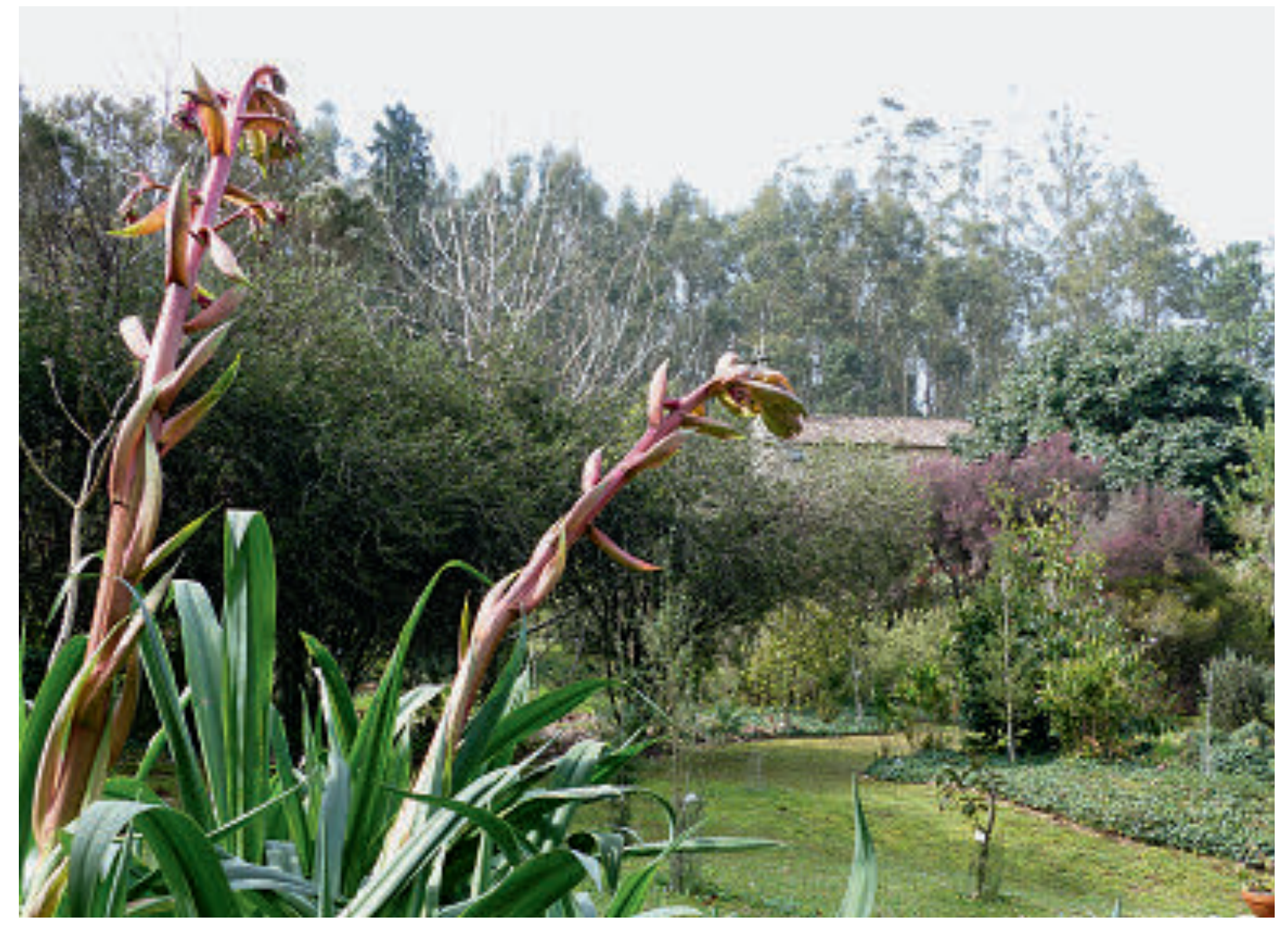

sodass der Besucher auf verwunschenen Pfaden oder auf der zentralen Wiese, manchmal auch ganz frei zwischen den Pflanzen, wandeln darf.

Gleich hinter dem Eingang befindet sich ein von den Gebäuden umgebener, geschützer Bereich. Hier gedeihen diverse Stauden. Im Frühling dominiert die Farbe Grün, denn man findet u.a. Pflanzen, die durch ihre attraktiven Blätter wirken wie z. B. panaschierte Agaven, eine noch sehr kleine Gunnera oder Philodendron. In der Scheune gibt es liebevolle Dekorationen aus getrockneten Kräutern. Zeugnis der landwirtschaftlichen Ausrichtung von Pazo da Saleta ist ein traditioneller Speicher (Horreo) für Feldfrüchte, Mais und Getreide. Dieser Typ von Speicher wird im Norden von Spanien noch recht häufig verwendet. Die Horreos ste-

Abb. 12 (Seite 116 oben): Der ehemalige Taubenturm.

Abb. 13 (Seite 116 unten): Banksia spinulosa mit ihren auffälligen orangegelben Blütenständen.

Abb. 14 (oben): Berschorneria yuccoides. hen frei und haben einen Unterbau aus Steinpfeilern. Der eigentliche Speicher besteht aus Holz oder Stein und enthält seitliche Lüftungsschlitze, da ohne sie in dem feuchten Klima Nordspaniens die Ernte verfaulen würde.

Ein schmaler Pfad entlang der Außenmauer des Hofes führt in den Kernbereich des Gartens, auf den sich von hier oben schöne Blicke ergeben. Im Frühling fallen aus der Ferne mehrere Magnolien (z. B. M. doltsopa, M. sprengeri, M. virginiana) auf. Gleich zu Beginn erscheinen viele Arten der Südhalbkugel, darunter verschiedene Myrtengewächse (z. B. Leptospermum, Eucalyptus), Akazien und Proteusgewächse (z. B. Grevillea rosmarinifolius, Telopea speciosissima, Protea cynaroides). Besonders auffällig ist eine kräftige Gruppe der in Südafrika heimischen Aloe plicatilis. Die stammbildende Art trägt an ihren Sprossenden jeweils einen Schopf aus ganz charakteristischen, wie ein Fächer angeordneten Blättern. In der natürlichen Heimat werden die roten, nektarreichen Blüten durch Vögel bestäubt. 

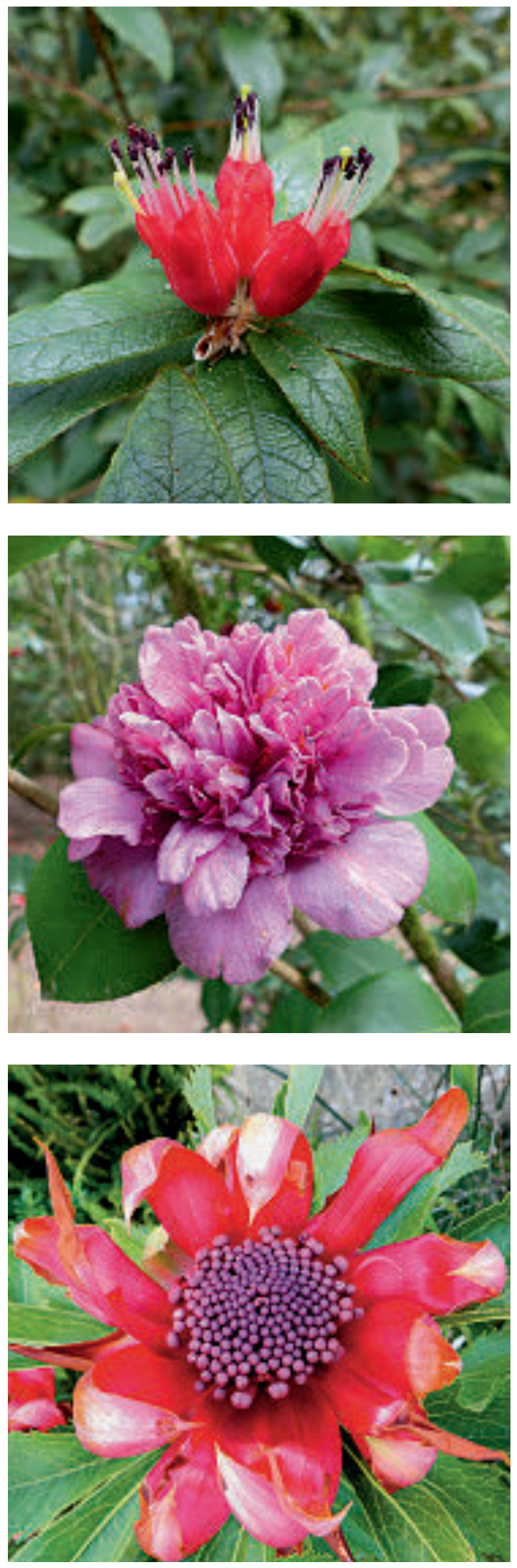

Mehr oder weniger im Zentrum des Gartens befindet sich ein achteckiger Taubenturm, in dem heute keine Tauben mehr leben, der dafür als Weinlager genutzt wird. Am Rande der benachbarten Wiese finden sich lauschige Sitzplätze. Es gibt hier attraktiv blühende Sträucher wie z. B. Illicium floridanum, ein Verwandter des Sternanis, mit leuchten roten Blüten. Drimys lanceolata wird auch Tasmanischer Pfeffer genannt, weil seine Früchte an Pfefferkörner erinnern, scharf schmecken und zum Würzen verwendet werden. Ein besonders attraktives Gehölz ist Banksia spinulosa mit seinen gelben, bürstenartigen Blütenständen.

Markant ist auch ein altes Exemplar eines Mexikanischen Erdbeerbaumes (Arbutus andrachnoides), dessen Rinde vor allem bei Regen leuchtend ziegelrot glänzt. Auch Beschorneria yuccoides stammt aus Mexiko und ist mit den Agaven verwandt. Schon im März bringen ihre auffälligen Blütenstände mit den grünen Blüten und roten Hochblättern Farbe in den Garten. Immer wieder stößt man auf nette dezente Dekorationen, und wenn es nur ein Arrangement aus Kamelienblüten und Zapfen auf dem Tisch einer Sitzgruppe ist.

Im Randbereich ist der Garten von größeren Gehölzen wie z. B. Kork- und anderen Eichen umgeben. Über die Jahre sind verwunschene schattige Bereiche mit Dschungelcharakter entstanden. Hier gedeihen große Kamelien und einige besondere Rhododendren. Unter letzteren begeistert $R h$. spinuliferum. Die aus China stammende Art bildet von Februar bis Juni ungewöhnliche röhrenförmige, rote Blüten, die von Vögeln bestäubt werden. Dieser Rhododen-

Abb. 15 (oben): Rhododendron spinuliferum mit für die Gattung ungewöhnlichen röhrenförmigen Blüten.

Abb. 16 (Mitte): Camellia japonica 'Dona Herzila da Freitas Magalhaes“.

Abb. 17 (unten): Telopea speciosissima wird in ihrer australischen Heimat von Vögeln bestäubt.

Abb. 18 (Seite 119 oben): Im Frühjahr stehen einige alte Rhododendren in voller Blüte.

Abb. 19 (Seite 119 unten): Herbstimpression im Gehölzbereich des Gartens. 


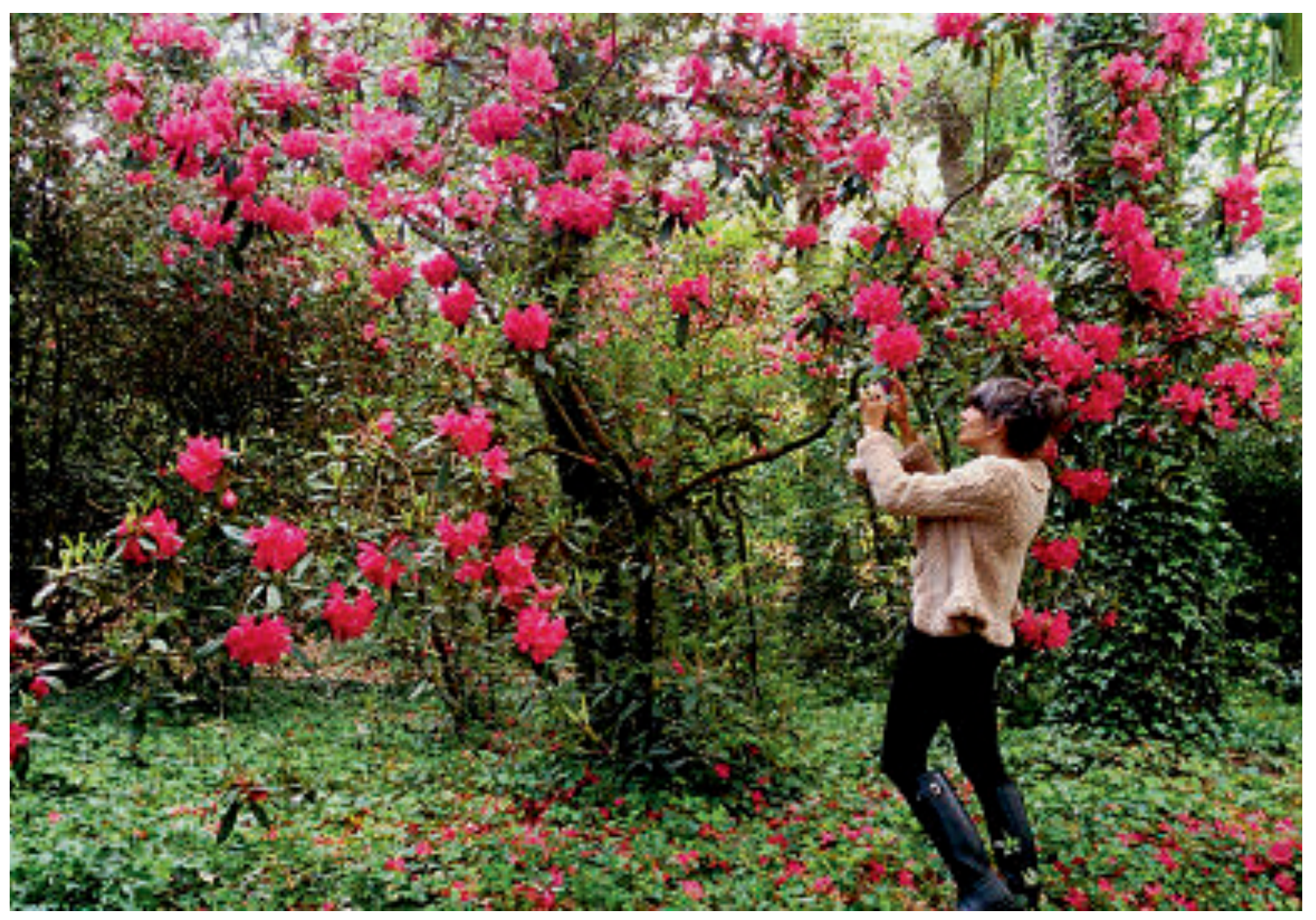

dron spielt in der traditionellen chinesischen Medizin eine Rolle bei der Behandlung von Asthma.

Aufgrund der großen Vielfalt im Garten lohnt sich ein Besuch fast während des ganzen Jahres, also nicht nur im zeitigen Frühjahr, wenn man ihn und andere Gärten wegen der Kamelien aufsucht. Auf einem gemütlichen Rundgang kann man eine Reise zu ausgewählten Pflanzen aus aller Welt unternehmen, ob es nun Watsonien und Pfauenblumen (Dietes grandiflora) aus Südafrika sind, Hardenbergia comptonianum und Leptospermum aus Australien, Lapageria rosea aus Chile oder Romneya coulteri aus Kalifornien sind. Der Park ist auch eine Oase für Tiere wie Insekten, Amphibien oder Vögel.

Eine Voranmeldung für den Besuch des Gartens ist notwendig. Nicht nur Botaniker kommen hier auf ihre Kosten. Im Garten werden auch verschiedene Events durchgeführt, er wird als Kulisse für Filmaufnahmen und Modeshootings zur Verfügung gestellt, die Kapelle ist für Trauungen nutzbar.

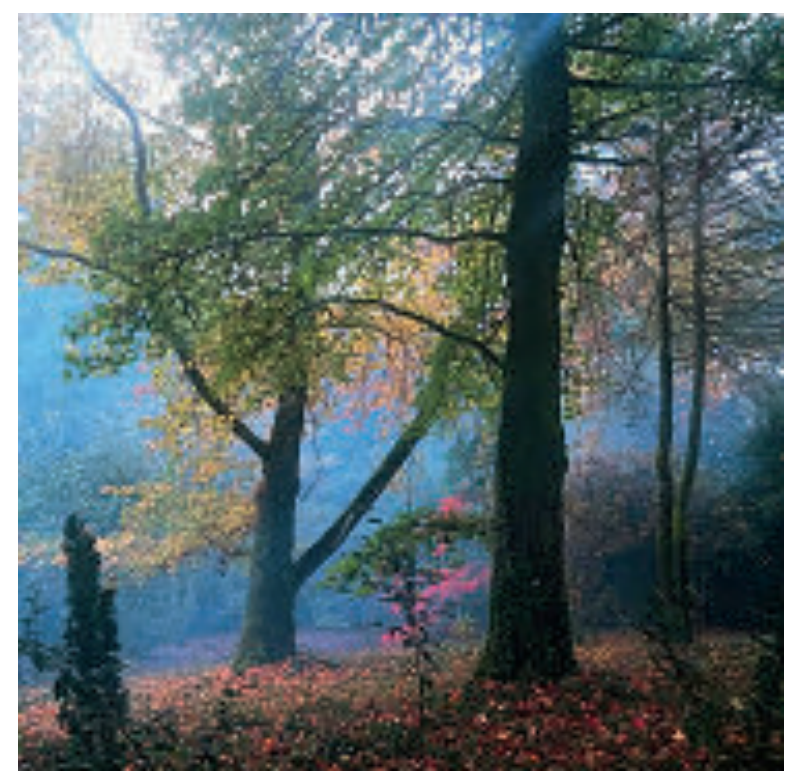

\section{Internetseiten}

http://www.pazodelasaleta.com/ http://www.cameliagalicia.com/saleta.html http://www.turismo.gal/portada-da-ruta-dacamelia?langId=es_ES 